\title{
Robust Control Strategy of Human Assistant Robot
}

\author{
Magdi S Mahmoud* \\ Systems Engineering Department, KFUPM, Saudi Arabia
}

Submission: June 10, 2017; Published: September 15, 2017

*Corresponding author: Magdi S Mahmoud, Systems Engineering Department, KFUPM Dhahran 31261, Saudi Arabia, Tel: 966-13-860-7516; Email: msmahmoud@kfupm.edu.sa

Abstract

Human assistant robot is a humanoid type robot which consists of a body with waist joint, arms designed for safety and a wheeled inverted pendulum mobile platform. The equation of motion is derived considering the non-holonomic constraint of the two-wheeled mobile robot with parameter uncertainties. A robust state feedback control method is applied for basic mobile controls wherein the control gain is calculated by linear quadratic control with proportional plus integral gain (LQRI). Through several experiments of balancing, linear running, and steering, it was confirmed that the robot could realize stable mobile motion in a real environment by the developed controller.

Keywords: Human assistant robot; Robust LQRI design; LMIs

\section{Introduction}

Recently, robots have been used in many applications including industry and human ordinary life. Robots can make the human life easier by doing the dangerous works such as working in a toxic environment or by doing the hard works such as carrying heavy objects. In addition, robots can add more precision and repeatability in many tasks such as welding and assembly tasks. Human assistant robot (HAR) is a humanoid type robot which consists of a body with waist joint, arms designed for safety, and a wheeled inverted pendulum mobile plat-form. Although the arms are designed at lowpower and lightweight for safety, it is capable to perform tasks that require high power by utilizing its self-weight, which is the feature of a wheeled inverted pendulum mobile platform.

HAR is modeled as a three dimensional robot; with controls of inclination angle, horizontal position, and steering angle to achieve high mobile capability. The motion equation is derived considering the non-holonomic constraint of the two-wheeled mobile robot. Wheeled inverted pendulum is considered highly unstable robot, thus achieving the stability is the key that controller approaches are designed for. Two wheeled mobile robot balancing controller has been tackled as a linearized model [1] or as a nonlinear model [2]. Another work tackled the nonlinear disturbance [3] by developing a nonlinear observer. In [4] a two wheeled mobile robot is designed and a comparison between the linearized and nonlinear model is done experimentally. An MPC controller resented by [5] was designed to maintain the robot at balance.
One of the key challenges in using robots is the uncertainty. This uncertainty can be in the robot parameters such as it mass, inertia and the friction factors or in the sensors readings such as the robot speed and inclination angle. And also uncertainty can be in the working area such as the carried object mass, inertia and size. The challenge is how to increase the performance of such robots with the existence of this uncertainty. By developing more robust and fast controller algorithms can tackle uncertainty [6-8].

In this paper, we consider the modeling and robust control design of the human assistant robot and generate an appropriate linearized model with parametric uncertainties. Then we develop a robust control design method based on linear quadratic control with integral term (LQRI) design and establish an LMI formalism yielding a proportional plus integral state-feedback gain. The design procedures are cast into the format of feasibility problem over linear matrix inequalities (LMIs). By this way, effective computational method is established yielding guaranteed quality solution. Simulation studies are performed on experimental setup and the ensuing results yield good performance $[9,10]$.

\section{Human Assistant Robot}

Figure 1 shows the case study HAR and is it consisting of two arms with one elbow for each arm which can be used to maintain stability of the robot in further research and two gyro sensors specified to define the acceleration and the inclination 
angle relative to the flat surface of the earth. Two wheels are for movements in two degree of freedom.

Table 1: Numerical Data

\begin{tabular}{|c|c|}
\hline Parameter & Value \\
\hline $\mathrm{M}_{\mathrm{g}}$ & $25.26 \mathrm{~kg}$ \\
\hline $\mathrm{m}_{\mathrm{g}}$ & $125 \mathrm{~kg}$ \\
\hline$\ell_{\mathrm{g}}$ & $0.4005 \mathrm{~m}$ \\
\hline $\mathrm{b}$ & $0.16 \mathrm{~m}$ \\
\hline$r_{w}$ & $0.1 \mathrm{~m}$ \\
\hline$I_{g}$ & $1.408 \mathrm{kgm}^{2}$ \\
\hline $\mathrm{I}_{\mathrm{wa}}$ & $0.01475 \mathrm{kgm}^{2}$ \\
\hline $\mathrm{I}_{\mathrm{wd}}$ & $0.0073 \mathrm{kgm}^{2}$ \\
\hline $\mathrm{I}_{\mathrm{ra}}$ & 0 \\
\hline $\mathrm{I}_{\mathrm{rd}}$ & 0 \\
\hline $\mathrm{g}$ & 1 \\
\hline$c_{r}$ & $0.1 \mathrm{Nms} / \mathrm{rad}$ \\
\hline $\mathrm{c}_{\ell}$ & $0.1 \mathrm{Nms} / \mathrm{rad}$ \\
\hline $\mathrm{G}$ & 9.81 \\
\hline
\end{tabular}

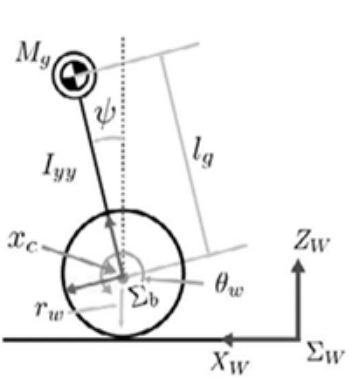

Figure 1: Two DOF human assistant robot.

A model of pendulum robot is built on two dimensional models with steering angle created from rotation and inclination angle caused by linear movements, see Figure 1. In addition, linear position of coordinates. The origin of vehicle coordinates $\Xi_{v}$ is located at the midpoint on the line that connects the axis of the two wheels, and the origin of the body coordinates $\Xi_{b}$ coincides with the $\Xi_{u}$. The angle between $z b$ and $z v$ represents the inclination angle of the body $\psi$, and the angle between $x_{v}$ and $X o$ axis of the global coordinates represents the steering angle. In all the coordinates, CCW direction is positive. The parameters in the model are described in Table 1 in the appendix.

Following the work of [11-16], the nonlinear robot model $x=f(x, u, w)$ can be conveniently cast into the uncertain linearized model:

$$
\begin{aligned}
x(\mathrm{t}) & =(\mathrm{A}+\delta \mathrm{A}) \mathrm{x}(\mathrm{t})+(\mathrm{B}+\delta \mathrm{B}) \mathrm{u}(\mathrm{t})+\Gamma \mathrm{w}(\mathrm{t}) \\
& =\mathrm{A}_{\delta} \mathrm{x}(\mathrm{t})+\mathrm{B}_{\delta} \mathrm{u}(\mathrm{t})+\Gamma \mathrm{w}(\mathrm{t}) \\
\mathrm{z}(\mathrm{t}) & =\mathrm{Gx}(\mathrm{t})+\operatorname{Du}(\mathrm{t})+\Phi \mathrm{w}(\mathrm{t}) \\
\mathrm{y}(\mathrm{t}) & =\mathrm{Cx}(\mathrm{t})+\Psi \mathrm{w}(\mathrm{t})
\end{aligned}
$$

Where $\quad x(t) \in \mathfrak{R}^{n}, u(t) \in \mathfrak{R}^{m}, y(t) \in \mathfrak{R}^{p} \quad$ are the state, the control input and the measured output vectors and $\delta A, \quad \delta B$ accounts for the parametric uncertainties and for convenience they are expr

$$
[\delta \mathrm{A} \delta \mathrm{B}]=\mathrm{H}_{\mathrm{a}} \mathrm{F}\left[\mathrm{E}_{\mathrm{a}} \mathrm{E}_{\mathrm{c}}\right], \mathrm{F}^{\mathrm{t}} \mathrm{F} \leq \mathrm{I}
$$

The state variables are $x_{v}, \Phi, \Psi, x_{v}, \dot{\Phi}, \Psi$ where $x_{v}$ the linear position of the vehicle (m) is $\Phi$ is the inclination angle of center of gravity (CoG) in (rad) $\psi$ and is the steering angle of the vehicle in ( $\mathrm{rad}$ ). The matrices $\mathrm{H}_{\mathrm{a}}, \mathrm{E}_{\mathrm{a}}, \mathrm{E}_{\mathrm{c}}$ are selected to reflect the amount of deviation in model parameters from nominal levels. Observe that modeling errors and/or parametric uncertainties are incorporated in (1) to reflect some practicality in replacing the nonlinear model $x=f(x, u, w)$. By quasi-linearization at appropriate operating model [11-13], the matrices $\mathrm{A}, \mathrm{B}, \mathrm{C}$ are given by:

$$
\begin{aligned}
& A=\left(\begin{array}{ll}
0 & I \\
A_{1} & A_{2}
\end{array}\right), B=\left(\begin{array}{l}
0 \\
B_{1}
\end{array}\right), C=\left(\begin{array}{ll}
I & 0
\end{array}\right) \\
& A_{1}=\left(\begin{array}{llc}
0 & 0 & a_{43} \\
0 & 0 & 0 \\
0 & 0 & a_{63}
\end{array}\right), A_{2}=\left(\begin{array}{lll}
a_{44} & a_{45} & a_{46} \\
a_{54} & a_{55} & a_{56} \\
a_{64} & a_{65} & a_{66}
\end{array}\right) \\
& B_{1}=\left(\begin{array}{ll}
b_{1} & b_{2} \\
b_{3} & b_{4} \\
b_{5} & b_{6}
\end{array}\right)
\end{aligned}
$$

Where the parameter definitions and values are given at the end.

\section{Control Design}

In control system terminology, the problem under consideration is that of determining feedback controller that makes system (1) stable over a wide range of operation while achieving a prescribed performance measure. Next, we provide design techniques to achieve this goal.

\section{LQRI: Proportional-integral gain}

A formulation of the performance criterion is considered which contains the standard linear-quadratic regulator (LQR) plus a term representing the integral of the deviation of the output from its initial state $z(t)=\int_{0}^{t} y(d \tau$. This formulation will be referred to as the LQRI.

$$
\left.\tilde{J}=\int_{0}^{\infty}\left(y(\tau)^{t} y(\tau)\right)+\rho u(\tau)^{t} u(\tau)+\sigma z(\tau)^{t} z(\tau)\right) d \tau
$$

Treating $z=C x$ as additional state variable, we define . $\eta=[x z]^{t}$ The augmented system becomes

$$
\begin{aligned}
& \dot{\eta}=\left(\begin{array}{ll}
A & 0 \\
0 & 0
\end{array}\right) \eta+\left(\begin{array}{l}
B \\
0
\end{array}\right) u \\
& =\tilde{A} \eta+\tilde{B} u \\
& \bar{y}=\left(\begin{array}{ll}
C & 0
\end{array}\right) \eta=\tilde{C} \eta
\end{aligned}
$$

And hence we can re-write (4) as:

$$
\begin{aligned}
& \tilde{J}=\int_{0}^{\infty}\left(y(\tau)^{t} y(\tau)+\rho u(\tau)^{t} u(\tau)+\sigma z(\tau)^{t}\right) d \tau . \\
& \tilde{Q}=\left(\begin{array}{cc}
C^{t} C & 0 \\
0 & \sigma I
\end{array}\right), \tilde{R}=\rho I, \rho, \sigma>0
\end{aligned}
$$


Next, we present an LMI-based formulation to the LQI control of system (5) while minimizing the quadratic cost (6). We proceed to determine a linear optimal control $u=\tilde{L} x$ that achieves this goal.

Assumption 1: There exists a Lyapunov functional $V(x)$ which has the properties:

- $\tilde{V}(\eta)=\eta^{t} \bar{P} \eta, \bar{P}>0$

- $\quad$ There exist $\gamma+>0$ such that $\eta_{o}^{t} \bar{P} \eta_{o} \leq \gamma+$

- $\quad \tilde{V}(\eta) \leq-\left[\eta^{t} Q \eta+\tilde{u^{t}} \tilde{R u}\right]$

The following theorem provides an LMI-based LQRI design for system (5) with linear control $u=\tilde{L} x$ :

Theorem 1: Given matrices $\tilde{Q}>0, \tilde{R}>0$ and scalar $\mathrm{n}>0$, system (5) $w(t) \equiv 0$ and the LQRI control $u=\tilde{L} x$ is robustly asymptotically stable for all admissible uncertainties $F^{t} F \leq I$ and $\tilde{J} \infty \leq \tilde{V}\left(\eta_{o}\right)$ if there exist matrices $S, Y>0$ such that

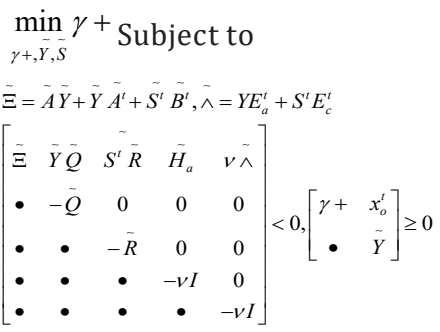

Has a feasible solution, then the proportional plus integral (PI) gain matrix is $L=S Y^{-1}$.

Proof: By Assumption (1), and using control $u=\tilde{L} \eta$ in system (5), the inequality of the derivative of the Lyapunov functional is expressed as

$$
\eta^{t}\left[\bar{P}(\bar{A}+\tilde{B} \bar{L})+(\bar{A}+\tilde{B} \tilde{L})^{t} \bar{P}\right] \eta \leq-\eta^{t}\left[\tilde{Q}+\tilde{L^{t}} \tilde{R} \tilde{L}\right] \eta
$$

It is evident that (8) is satisfied if there exists and such that

$$
\left[\bar{P}(\vec{A}+\bar{B} \bar{L})+(\bar{A}+\bar{B} \bar{L})^{t} \bar{P}\right]-\left[\bar{Q}+\overrightarrow{L^{t}} \bar{R} \bar{L}\right] \leq 0
$$

Simple computations on (6) in view of Assumption (1) yields $J \infty \leq V\left(\eta_{o}\right)$. By minimizing the upper bound $\gamma+$ on the cost $\eta_{o}^{t} \stackrel{-}{P} \eta_{o}$ we obtain

$$
\min \gamma+\text { Subject to }
$$

To convexify the above problem, we define

$$
\tilde{H}_{a}=\left[\begin{array}{c}
H_{a} \\
0
\end{array}\right], \tilde{E}_{a}^{t}=\left[\begin{array}{c}
E_{a}^{t} \\
0
\end{array}\right], \tilde{E}_{c}^{t}=\left[\begin{array}{c}
E_{c}^{t} \\
0
\end{array}\right]
$$

$$
\text { and express (9) as }
$$

$$
\begin{aligned}
\tilde{\Phi} & =\tilde{P}(\tilde{A}+\tilde{B} \tilde{L})+(\tilde{A}+\tilde{B} \tilde{L})^{t} \bar{P} \\
\tilde{\Psi} & =\tilde{E}_{a}+\tilde{E_{a}} \tilde{L} \\
\tilde{\Pi} & =\left[\begin{array}{cccccc}
\tilde{\Phi} & \tilde{Q} & \tilde{L^{t}} \tilde{R} & \bar{P} \tilde{H}_{a} & v \tilde{\Psi}^{\prime} \\
\bullet & -\tilde{Q} & 0 & 0 & 0 \\
\bullet & \bullet & -\tilde{R} & 0 & 0 \\
\bullet & \bullet & \bullet & -v I & 0 \\
\bullet & \bullet & \bullet & \bullet & -v I
\end{array}\right]<0
\end{aligned}
$$

Pre- and post-multiply (12) by diag $\{\tilde{Y}, I, I, I, I\}$ and using $\tilde{Y}=P^{-1}, \tilde{S}=\tilde{L} P^{-1}$ it yields (7). Additionally, the inequality bound of the Lyapunov functional can be expressed as

$\left[\begin{array}{cc}\gamma+ & \eta_{o}^{t} \\ & - \\ \bullet & P^{-1}\end{array}\right] \geq 0$

This can be manipulated to yield the other part of (7). When a feasible solution is attained, we get, $\tilde{L}=\tilde{S} Y^{-1}$ which yields the PI gain matrix as desired.

\section{Simulation Results}

The expressions of the different parameters are given below:

$$
\begin{aligned}
& a_{43}=\frac{M_{g}^{2} \ell_{g}^{2} r_{w}^{2} G}{V_{1}}, a_{44}=\frac{w_{1} c_{1}}{V_{1}}, a_{55}=\frac{-b^{2} c_{1}}{V_{2}} \\
& a_{45}=\frac{w_{1} c_{1}}{V_{1}}, a_{46}=\frac{-r_{w} w_{1} c_{1}}{V_{1}}, a_{54}=\frac{-b c_{2}}{V_{2}}, \\
& a_{56}=\frac{b r_{w} w_{1} c_{1}}{V_{2}}, a_{64}=\frac{-w_{2} c_{1}}{r_{w} V_{1}}, a_{65}=\frac{-b w_{2} c_{2}}{r_{w} V_{1}}, \\
& a_{63}=\frac{\left(M_{g} r_{w}^{2}+2 m_{w} r_{w}^{2}+2\left(I_{w a}+\gamma I_{r a}\right)\right) M_{g} I_{g} G}{V_{1}} \\
& b_{1}=b_{2}=\frac{-r_{w} w_{1}}{V_{1}}, b_{3}=-b_{4}=\frac{-r_{w} b}{V_{2}} \\
& b_{5}=b_{6}=\frac{w_{2}}{V_{1}}, a_{66}=\frac{-w_{2} c_{1}}{V_{1}}, \\
& V_{1}=I_{z 2} r_{w}^{2}+2\left(I_{w d}+I_{r d}\right)+2 m_{w} r_{w}^{2} b^{2}+2\left(I_{w a}+\gamma^{2} I_{r a}\right) b^{2}, w_{1}=M_{g} \ell_{g}^{2}+I_{y y}+M_{g} \ell_{g} r_{w} \\
& w_{2}=M_{g} \ell_{g} r_{w}+M_{g} r_{w}^{2}+2 m_{w} r_{w}^{2}+2\left(I_{w a}+\gamma^{2} I_{r a}\right)
\end{aligned}
$$

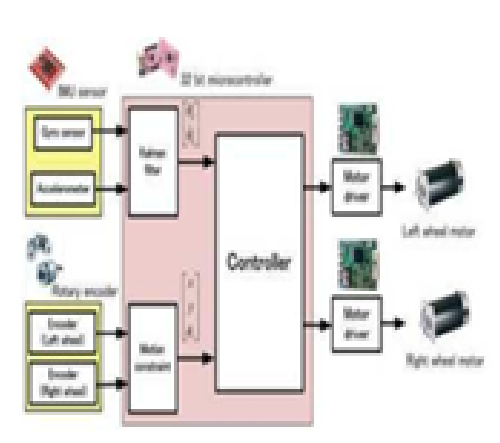

Figure 2: Human assistant robot experiment.

The values of the basic elements are presented in Table I Using the lab capabilities at KFUPM, Figure 2 illustrates twowheeled inverted pendulum robot experiment. It consists of two motor drivers, two motor, a 32-bit microcontroller, an inertial measurement unit (IMU), and two encoders. 3-axis gyro sensor and 3-axis accelerometer are installed in IMU sensor. To operate the robot as shown in Figure 2, IMU sensor precedes the data of gyro sensor and accelerometer by Kalman filter in order to estimate the angular velocity and tilt angle of the body. The encoder is detected the rotation of both wheels then they are converted to position and velocity in Cartesian coordinate frame. Then, position, velocity, yaw angle, yaw rate, tilt angle, pitch rate are used to compute by control law. It will 


\section{Robotics \& Automation Engineering Journal}

send the command to motor drivers in order that the motor drivers produce electronic current and voltage for motors.

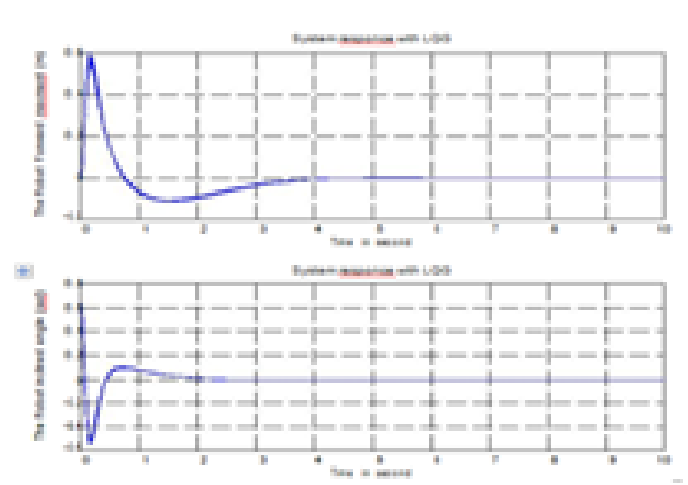

Figure 3: Closed-loop response using LQRI controller.

Several simulation experiments of balancing, linear running, and steering have been carried out using the stabilization method LQRI. The uncertainties are added to the mass and inertia of the robot. $\pm 10 \%$ of it nominal values. The ensuing results are depicted in (Figure 3), from which it was confirmed that the robot could realize stable mobile motion in a real environment by the developed controller.

\section{Conclusion}

This article introduced the linearized dynamic model of two wheeled inverted pendulum robot subject to uncertain parameters, robust control design and an experimental result. The control technique focused on linear quadratic methods with proportional plus integral using linear-matrix inequalities. Improved analytical results are developed and simulation results are presented.

\section{Acknowledgment}

This research work is supported by the deanship of scientific research (DSR) at KFUPM through distinguished professorship project no. IN 141003.

\section{References}

1. Jeong S, Takahashi T (2008) Wheeled inverted pendulum typ e assistant robot: design concept and mobile control," Intell. Service Robot 1(4): 313-320.

2. Sinha A, Prasoon P, Bharadwaj PK, Ranasinghe AC (2015) Nonlin-ear Autonomous Control of a Two-wheeled inverted pendulum mobile robot based on Sliding Mode. Int. Conference Computational Intelligence and Networks (CINE) pp. 52-57.
3. Huang J, Ri S, Liu L, Wang Y, Kim J, Pak G (2015) Nonline ar Dis-turbance Observer-Based Dynamic Surface Control of Mobile Wheeled Inverted Pendulum. IEEE Trans. Control Syst. Technol 23(6): 2400-2407.

4. Garzn MAH, Hernndez WOC, Reyes APG, Quintero OEC (2015) Two-Wheeled Inverted Pendulum Robot NXT Lego Mindstorms: Mathematical Modelling and Real Robot Comparisons. Rev Politcnica 36(1).

5. Azimi MM, Koofigar HR (2013) Model predictive control fo $r$ a two wheeled self balancing robot, First RSI/ISM Int. Conference Robotics and Mechatronics (ICRoM) pp. 152-157.

6. Li Z, Xu C (2009) Adaptive fuzzy logic control of dynamic ba lance and motion for wheeled inverted pendulums. Fuzzy Sets Syst 160(12): 1787-1803.

7. Jung S, Kim SS (2008) Control Experiment of a Wheel-Driven M obile Inverted Pendulum Using Neural Network. IEEE Trans. Control Syst Technol 16(2): 297-303.

8. Huang J, Guan ZH, Matsuno T, Fukuda T, Sekiyama K (2010) SlidingMode Velocity Control of Mobile-Wheeled Inverted -Pendulum Systems. IEEE Trans Robot 26(4): 750-758.

9. Jeong S, Takahashi T (2007) Wheeled inverted pendulum typ e assistant robot: inverted mobile, standing, and sitting motions. IEEE/RSJ International Conference on Intelligent Robots and Systems pp. 19321937.

10. Mahmoud MS (2004) Resilient control of uncertain dynamical systems, Springer, Heidelberg.

11. Jeong S, Takahashi $\mathrm{T}$ (2008) Wheeled inverted pendulum t ype assistant robot: design concept and mobile control," Intelligent Service Robotics 1(4): 313-320.

12. Sinha A, Prasoon P, Bharadwaj PK, Ranasinghe AC (2015) Nonlinear autonomous control of a two-wheeled inverted pendulum mobile robot based on sliding mode. Int. Conference on Computational Intelligence and Networks (CINE) pp. 52-57.

13. Huang J, S Ri, Liu L, Wang Y, Kim J, et al. (2015) Nonlinear disturbance observer-based dynamic surface control of mobile wheeled inverted pendulum. IEEE Trans. Control Syst. Technol 23(6): 2400-2407.

14. Garzn MAH, Hernndez WOC, Reyes APG, Quin-tero OEC (2015) Two-wheeled inverted pendulum robot NXT Lego Mindst orms: mathematical modeling and real robot comparisons. Rev Politcnica 36(1): 2015.

15. Azimi MM, Koofigar HR (2013) Model predictive control f or a two wheeled self balancing robot. First RSI/ISM Int. Conference Robotics and Mechatronics (ICRoM) pp.152-157.

16. Pathak K, Franch J, Agrawal SK (2005) Velocity and position control of a wheeled inverted pendulum by partial feedback linearization, IEEE Trans Robotics 21(3): 505-512. 


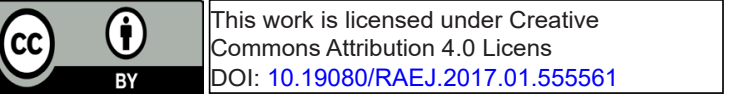

Your next submission with Juniper Publishers will reach you the below assets

- Quality Editorial service

- Swift Peer Review

- Reprints availability

- E-prints Service

- Manuscript Podcast for convenient understanding

- Global attainment for your research

- Manuscript accessibility in different formats ( Pdf, E-pub, Full Text, Audio)

- Unceasing customer service

Track the below URL for one-step submission https://juniperpublishers.com/online-submission.php 